IJMMS 2003:69, 4363-4371

PII. S0161171203203471

http://ijmms.hindawi.com

(c) Hindawi Publishing Corp.

\title{
EXTENSIONS OF RATIONAL MODULES
}

\author{
J. CUADRA
}

Received 30 March 2002

\begin{abstract}
For a coalgebra $C$, the rational functor Rat $(-): \mu_{C^{*}} \rightarrow \mu_{C^{*}}$ is a left exact preradical whose associated linear topology is the family $\mathscr{F}_{C} C$, consisting of all closed and cofinite right ideals of $C^{*}$. It was proved by Radford (1973) that if $C$ is right $\mathscr{F}^{*}$ Noetherian (which means that every $I \in \mathscr{F}_{C} C$ is finitely generated), then $\operatorname{Rat}(-)$ is a radical. We show that the converse follows if $C_{1}$, the second term of the coradical filtration, is right $\mathscr{F}$-Noetherian. This is a consequence of our main result on $\mathscr{F}$ Noetherian coalgebras which states that the following assertions are equivalent: (i) $C$ is right $\mathscr{F}$-Noetherian; (ii) $C_{n}$ is right $\mathscr{F}$-Noetherian for all $n \in \mathbb{N}$; and (iii) $\mathscr{F}_{C}$ is closed under products and $C_{1}$ is right $\mathscr{F}$-Noetherian. New examples of right $\mathscr{F}$ Noetherian coalgebras are provided.
\end{abstract}

2000 Mathematics Subject Classification: 16W30, 18E40.

1. Introduction. Let $C$ be a coalgebra over a field $k$ and $C^{*}$ its dual algebra. Let ${ }^{C} \mathcal{M}$ denote the category of left $C$-comodules and $\mu_{C^{*}}$ the category of right $C^{*}$-modules. It is well known that ${ }^{C} \mathcal{M}$ is isomorphic to the subcategory $\operatorname{Rat}\left(M_{C^{*}}\right)$ of all rational right $C^{*}$-modules. Indeed, $\operatorname{Rat}\left(M_{C^{*}}\right)$ is a hereditary pretorsion class in $M_{C^{*}}$ (i.e., a class closed under subobjects, quotients, and arbitrary direct sums). The linear topology $\mathscr{F}_{C}$ on $C^{*}$ associated to $\operatorname{Rat}\left(\mu_{C^{*}}\right)$ consists of all closed and cofinite right ideals of $C^{*}$. The problem of when $\operatorname{Rat}\left(\mu_{C^{*}}\right)$ is a torsion class (closed under extensions) has been studied in $[5,6,8,10]$ and, recently, in [2]. In this paper, we continue the study of this problem and relate it to a finiteness condition on $\mathscr{F}_{C}$, that every $I \in \mathscr{F}_{C}$ is finitely generated. Coalgebras satisfying this property are called right $\mathscr{F}$-Noetherian. It was proved in

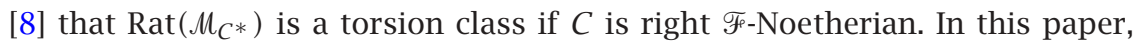
we find a hypothesis under which the converse holds. This is a consequence of our main theorem (Theorem 3.11) on $\mathscr{F}$-Noetherian coalgebras that allows to lift the property of being $\mathscr{F}$-Noetherian through the terms of the coradical filtration. It states that $C$ is right $\mathscr{F}_{F}$-Noetherian if and only if each term $C_{n}$ of

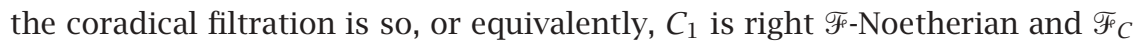
is closed under products. From this theorem, it follows that, for $C_{1}$ being right $\mathscr{F}$-Noetherian, $\operatorname{Rat}\left(M_{C^{*}}\right)$ is a torsion class if and only if $C$ is right $\mathscr{F}$-Noetherian. The hypothesis of $C_{1}$ being right $\mathscr{F}$-Noetherian is investigated in several cases. When $C$ is almost connected, $C_{1}$ is right $\mathscr{F}$-Noetherian if and only if $C_{1}$ is finite dimensional. If $C$ is pointed, $C_{1}$ is right $\mathscr{F}$-Noetherian if and only if, for every 
group-like element $x \in G(C)$, the set $\left\{\operatorname{dim}\left(P_{x, y}(C)\right): y \in G(C)\right\}$ is bounded. Finally, as another consequence of the main theorem, we provide new examples of right $\mathscr{F}$-Noetherian coalgebras. These are constructed by imposing the terms of the coradical filtration to be left semiperfect.

2. Preliminary results. Recall the following facts on torsion theories and coalgebras that will be needed in the sequel.

TORSION THEORY. Our main reference is [11, Chapter VI]. Let $R$ be a ring and $\mu_{R}$ the category of right $R$-modules. A class $\mathscr{C} \subset M_{R}$ is called a hereditary pretorsion class if it is closed under subobjects, quotients, and direct sums. If, in addition, $\mathscr{C}$ is closed under extensions, then it is called a torsion class. A functor $r: \mathcal{M}_{R} \rightarrow \mathcal{M}_{R}$ is called a left exact preradical if it is an idempotent left exact subfunctor of the identity functor of $\mu_{R}$. Such a functor is called a radical if $r(M / r(M))=\{0\}$ for all $M \in M_{R}$. A right linear topology on $R$ is a family $\mathcal{T}$ of right ideals of $R$ satisfying the following:

(T1) if $I \in \mathscr{T}$ and $I \subset J$, then $J \in \mathscr{T}$;

(T2) if $I, J \in \mathscr{T}$, then $I \cap J \in \mathscr{T}$;

(T3) for any $I \in \mathcal{T}$ and $a \in R$, the right ideal $(I: a) \in \mathcal{T}$.

A Gabriel topology is a right linear topology $\mathscr{T}$ satisfying the additional axiom

(T4) if $I$ is a right ideal of $R$ and there is $J \in \mathscr{T}$ such that $(I: b) \in \mathscr{T}$ for all $b \in J$, then $I \in \mathscr{T}$.

There is a bijective correspondence between

(i) right linear topologies on $R$,

(ii) hereditary pretorsion classes in $\mu_{R}$,

(iii) left exact preradicals in $\mu_{R}$.

Given a linear topology $\mathscr{T}$, the associated hereditary pretorsion class is $\mathscr{C}=$ $\left\{M \in \mathcal{M}_{R} \mid \operatorname{Ann}(m) \in \mathcal{T}\right.$ for all $\left.m \in M\right\}$. The associated left exact preradical $r$ is defined as follows: for any $M \in \mathcal{M}_{R}, r(M)=\{m \in M: \operatorname{Ann}(m) \in \mathscr{T}\}$. Conversely, if $\mathscr{C}$ is a hereditary pretorsion class, the corresponding linear topology $\mathcal{T}$ consists of all right ideals $I$ of $R$ for which $R / I \in \mathscr{C}$. The associated left exact preradical is defined in the following way: for any $M \in M_{R}, r(M)$ is the sum of all submodules of $M$ belonging to $\mathscr{C}$. This correspondence becomes a bijective correspondence between Gabriel topologies, torsion classes, and radicals.

COALgebras AND comodules. Throughout all vector spaces, algebras, coalgebras, $\otimes$, and so forth, are over a fixed ground field $k$. For general facts on coalgebras and comodules, we refer to $[1,7,12]$. For a coalgebra $C$, its dual algebra $C^{*}$ is a topological vector space with the weak-* topology. The closed subspaces of $C^{*}$ are the annihilators $W^{\perp\left(C^{*}\right)}$ of subspaces $W$ of $C$. A subspace $U$ of $C^{*}$ is called cofinite if $C^{*} / U$ is of finite dimension. A right ideal $J$ of $C^{*}$ is closed and cofinite if and only if there is a finite-dimensional right coideal $W$ of $C$ such that $J=W^{\perp\left(C^{*}\right)}$.

The category ${ }^{C} \mathcal{M}$ of left $C$-comodules is isomorphic to the full subcategory $\operatorname{Rat}\left(\mu_{C^{*}}\right)$ of $\mu_{C^{*}}$ consisting of all rational right $C^{*}$-modules. Let $M \in \mu_{C^{*}}$ and 
$m \in M$. We say that $m$ is a rational element if there is $\rho_{m}=\sum_{i} c_{i} \otimes m_{i} \in C \otimes M$ such that

$$
m \cdot c^{*}=\sum_{i=1}^{n}\left\langle c^{*}, c_{i}\right\rangle m_{i} \quad \forall c^{*} \in C^{*} .
$$

The set consisting of all rational elements of $M$, denoted by $\operatorname{Rat}(M)$, is a $C^{*}$ submodule of $M$. When $M=\operatorname{Rat}(M), M$ is called rational. The assignment $\operatorname{Rat}_{C}(-): M_{C^{*}} \rightarrow M_{C^{*}}, M \mapsto \operatorname{Rat}(M)$, called the rational functor, is a left exact preradical. The hereditary pretorsion class associated to this preradical is the subcategory $\operatorname{Rat}\left(M_{C^{*}}\right)$ of all rational left $C^{*}$-modules. The linear topology $\mathscr{F}_{C}$ corresponding to this class is the family of all closed cofinite right ideals of $C^{*}$. It follows from the fundamental theorem on coalgebras that $\mathscr{F}_{C}$ is a symmetric linear topology. This means that, for every $J \in \mathscr{F}_{C}$, there is a two-sided ideal $K$ of $C^{*}$ such that $K \in \mathscr{F}_{C}$ and $K \subset J$.

\section{When is the rational functor a radical?}

DEFINITION 3.1. A coalgebra $C$ is said to have a right torsion rat functor if it satisfies one of the following equivalent conditions:

(i) $\operatorname{Rat}\left(\mu_{C^{*}}\right)$ is closed under extensions;

(ii) the rational functor is a radical;

(iii) $\mathscr{F}_{C}$ is a Gabriel topology.

It was proved in [5, Proposition 4 and Theorem 6] that these coalgebras enjoy the following properties.

Proposition 3.2. Having a right torsion rat functor is closed under subcoalgebras and arbitrary direct sums.

We give a necessary condition to have a torsion rat functor. We recall from [4] that a coalgebra $C$ is locally finite if $D \wedge_{C} D$ is finite dimensional for any finitedimensional subcoalgebra $D$, where $\wedge_{C}$ denotes the wedge product over $C$. See $[12$, Section 9.0] for the definition of the wedge product and its properties.

LEMMA 3.3. Let $C$ be a coalgebra such that $\mathscr{F}_{C}$ is closed under products. Then $C$ is locally finite.

Proof. Let $D$ be a finite-dimensional subcoalgebra of $C$. By hypothesis, there is a finite-dimensional subspace $W$ of $C$ such that $D^{\perp\left(C^{*}\right)} D^{\perp\left(C^{*}\right)}=W^{\perp\left(C^{*}\right)}$. Now, $D \wedge_{C} D=\left(D^{\perp\left(C^{*}\right)} D^{\perp\left(C^{*}\right)}\right)^{\perp(C)}=W^{\perp\left(C^{*}\right) \perp(C)}=W$. Hence, $D \wedge_{C} D$ is of finite dimension.

The converse of Lemma 3.3 is not true. A counterexample may be found in [9, Example 3.4]. Since any Gabriel topology is closed under products [11, Lemma 5.3], we have the following corollary. 
COROLLARY 3.4. Any coalgebra having a right torsion rat functor is locally finite.

A sufficient condition to have a torsion rat functor was given in [8, page 521].

Proposition 3.5. Let $C$ be a coalgebra such that every $J \in \mathscr{F}_{C}$ is finitely generated. Then $C$ has a right torsion rat functor.

DEFINITION 3.6. A coalgebra $C$ satisfying the hypothesis of Proposition 3.5 is called right $\mathscr{F}$-Noetherian.

EXAMPLE 3.7. (i) Let $A$ be an algebra such that every cofinite right ideal of $A$ is finitely generated. It follows from [4, Theorem 3.3] that the finite dual $A^{o}$ is right $\mathscr{F}$-Noetherian. In particular, the dual of a finitely generated algebra is right and left $\mathscr{F}$-Noetherian.

(ii) Recall from [6] that a coalgebra $C$ is called left semiperfect if the injective hull of any simple left $C$-comodule is finite dimensional. It was proved in [2, Theorem 2.12] that any left semiperfect coalgebra is right $\mathscr{F}$-Noetherian.

(iii) Subcoalgebras and arbitrary direct sums of right $\mathscr{F}$-Noetherian coalgebras are also right $\mathscr{F}$-Noetherian, see [10, Corollary 4.9] and [2, Proposition 2.8].

Our next step is to find out some structural properties of right $\mathscr{F}$-Noetherian coalgebras. Recall from [13, Example 1.2] that a right $C$-comodule $M$ is said to be finitely cogenerated if there is an injective $C$-comodule map $f: M \rightarrow C^{(n)}$ for some $n \in \mathbb{N}$. For any $M \in M^{C}$, let $E(M)$ denote its injective hull.

Proposition 3.8. Let I be a right coideal of $C$. The following assertions are equivalent:

(i) $C / I$ is finitely cogenerated;

(ii) $E(I) / I$ is finitely cogenerated;

(iii) $I^{\perp\left(C^{*}\right)}$ is a finitely generated right ideal of $C^{*}$.

Proof. (i) $\Leftrightarrow$ (ii). It is just to take into account that $C \cong E(I) \oplus T$ for some subcomodule $T$ of $C$ and $C / I \cong E(I) / I \oplus T$, see [3, Theorem $1.5 \mathrm{~g}$ ].

(i) $\Rightarrow$ (iii). Notice that $(C / I)^{*} \cong I^{\perp\left(C^{*}\right)}$ as right $C^{*}$-modules. Since $C / I$ is finitely cogenerated, there is an injective $C$-comodule map $f: C / I \rightarrow C^{(n)}$ for some $n \in \mathbb{N}$. The dual map $f^{*}: C^{*(n)} \rightarrow(C / I)^{*} \cong I^{\perp\left(C^{*}\right)}$ is a surjective $C^{*}$-module map. Thus, $I^{\perp\left(C^{*}\right)}$ is finitely generated.

(iii) $\Rightarrow\left(\right.$ i). We can express $C^{(n)}=W \otimes C$ with $W$ a space of dimension $n$. Let $\left\{w_{i}\right\}_{i=1}^{n}$ be a basis of $W$ and $\left\{w_{i}^{*}\right\}_{i=1}^{n}$ its dual basis in $W^{*}$. By hypothesis, there is a surjective $C^{*}$-module map $f: W^{*} \otimes C^{*} \rightarrow I^{\perp\left(C^{*}\right)}$. Let $c_{i}^{*}=f\left(w_{i}^{*} \otimes \varepsilon\right)$ for all $i=1, \ldots, n\left(\varepsilon\right.$ is the counit of $C$ ). We define $g: C \rightarrow C^{(n)}$ by $g(c)=\sum_{i=1}^{n} w_{i} \otimes$ $\left(\sum_{(c)}\left\langle c_{i}^{*}, c_{(1)}\right\rangle c_{(2)}\right)$ for all $c \in C$. It is easy to check that $g^{*}=f$. Hence, $g$ is a $C$-comodule map. Moreover, $I^{\perp\left(C^{*}\right)}=\operatorname{Im}\left(g^{*}\right)=\operatorname{Ker}(g)^{\perp\left(C^{*}\right)}$. Then $I=\operatorname{Ker}(g)$. Therefore, $C / I$ is finitely cogenerated. 
In view of the preceding result, the closed right ideals of $C^{*}$ are finitely generated if the quotients of $C$ are finitely cogenerated as a right comodule. For a locally finite coalgebra, this latter property may be characterized by studying the socle of such a quotient. It is known that there is a bijective correspondence between simple subcoalgebras of $C$ and isomorphism classes of simple right $C$ comodules, see [1, Theorem 3.1.4]. Let $\mathscr{Y}$ denote the set of simple subcoalgebras of $C$. For each $D \in \mathscr{Y}$, let $S_{D}$ be the corresponding simple right comodule. It is also known that the socle of $C$ as a right comodule coincides with the coradical of $C$ and $C_{0}$ and it decomposes as $C_{0}=\operatorname{soc}(C) \cong \oplus_{D \in S} S_{D}^{\left(n_{D}\right)}$, where the $n_{D}$ are natural numbers, see [3, Theorem 1.3b]. For any right $C$-comodule $M, \operatorname{soc}(M) \cong$ $M \square_{C} C_{0}$. The isotypic component of $S_{D}$ in $M$ is given by $\rho^{-1}\left(M \square_{C} D\right)$, where $\rho: M \rightarrow M \otimes C$ is the structure map of $M$.

The following technical lemma will be useful to describe the simple comodules appearing in $\operatorname{soc}(C / I)$ for a right coideal $I$ of $C$.

LEMMA 3.9. Let $I$ be a right coideal of $C$ and $E$ a subcoalgebra of $C$. Then, $\left(I \wedge_{C} E\right) / I \cong(C / I) \square_{C} E$ as right $E$-comodules.

Proof. It is not difficult to verify that the map

$$
\Phi: I \wedge_{C} E \longrightarrow \frac{C}{I} \square_{C} E, \quad c \longmapsto \sum_{(c)}\left(c_{(1)}+I\right) \otimes C_{(2)},
$$

is a surjective $E$-comodule map whose kernel is $I$.

LEMMA 3.10. Let $C$ be a locally finite coalgebra and I a right coideal of finite dimension. Then $C / I$ is finitely cogenerated if and only if there is $\gamma \in \mathbb{N}$ such that $\operatorname{dim}\left(\left(I \wedge_{C} D\right) / I\right) \leq \gamma n_{D} \operatorname{dim}\left(S_{D}\right)$ for every $D \in \mathscr{Y}$.

Proof. Using Lemma 3.9, we have that

$$
\operatorname{soc}\left(\frac{C}{I}\right) \cong \frac{C}{I} \square_{C} C_{0} \cong \oplus_{D \in \mathcal{S}} \frac{C}{I} \square_{C} D \cong \oplus_{D \in \mathcal{S}} \frac{I \wedge_{C} D}{I} .
$$

Each $\left(I \wedge_{C} D\right) / I \cong S_{D}^{\left(m_{D}\right)}$ and $m_{D}$ is finite because $C$ is locally finite. Notice that $C / I$ is finitely cogenerated if and only if $\operatorname{soc}(C / I)$ is finitely cogenerated as a $C_{0}$-comodule. This happens if and only if there is $\gamma \in \mathbb{N}$ such that $m_{D} \leq \gamma n_{D}$ for all $D \in \mathscr{Y}$. Equivalently, $\operatorname{dim}\left(\left(I \wedge_{C} D\right) / I\right)=m_{D} \operatorname{dim}\left(S_{D}\right) \leq \gamma n_{D} \operatorname{dim}\left(S_{D}\right)$.

This description of the socle using the wedge yields a method to lift the

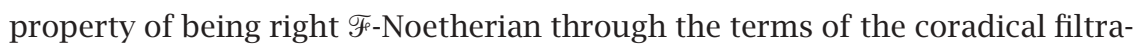
tion.

THEOREM 3.11. Let $\left\{C_{n}\right\}_{n \in \mathbb{N}}$ be the coradical filtration of $C$. The following assertions are equivalent:

(i) $C$ is right $\mathscr{F}$-Noetherian;

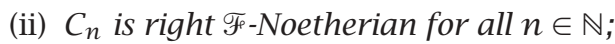

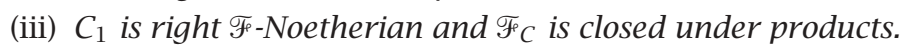




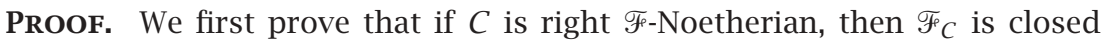
under products. Let $J, K \in \mathscr{F}_{C}$. By hypothesis, $J, K$ are finitely generated. Let $H$ be a two-sided ideal such that $H \in \mathscr{F}_{C}$ and $H \subseteq J$. Again by hypothesis, $H$ is finitely generated as a right ideal. By [4, Lemma 1.1.1], $K H$ is finitely generated and cofinite. Since it is finitely generated, it is closed by [4, Proposition 1.3.1(b)]. Hence, $K H \in \mathscr{F}_{C}$. From $K H \subset K J$, it follows that $K J \in \mathscr{F}_{C}$.

(i) $\Rightarrow$ (ii) and (i) $\Rightarrow$ (iii) follow from the fact that being right $\mathscr{F}$-Noetherian is closed under subcoalgebras.

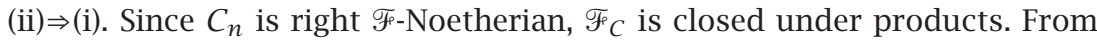
Lemma $3.3, C_{n}$ is locally finite for all $n \in \mathbb{N}$. In particular, $C_{1}$ is locally finite. By [4, Proposition 2.4.5], $C$ is locally finite.

Let $I$ be a finite-dimensional right coideal of $C$. In view of Proposition 3.8, it suffices to prove that $C / I$ is finitely cogenerated. There is $m \in \mathbb{N}$ such that $I \subset$ $C_{m}$. By hypothesis and Proposition 3.8, $C_{m+1} / I$ is finitely cogenerated. Applying Lemma 3.10, there is $\gamma \in \mathbb{N}$ such that $\operatorname{dim}\left(\left(I \wedge_{C_{m+1}} D\right) / I\right) \leq \gamma n_{D} \operatorname{dim}\left(S_{D}\right)$ for every $D \in \mathscr{S}$. On the other hand, $I \wedge_{C} D \subset C_{m} \wedge_{C} C_{0}=C_{m+1}$. By [4, Section 2.3.4], $I \wedge_{C} D=I \wedge{ }_{C_{m+1}} D$. Then, $\operatorname{dim}\left(\left(I \wedge_{C} D\right) / I\right) \leq \gamma n_{D} \operatorname{dim}\left(S_{D}\right)$ for each $D \in \mathscr{Y}$. Lemma 3.10 implies that $C / I$ is finitely cogenerated.

(iii) $\Rightarrow$ (i). We first check that every closed and cofinite maximal ideal is finitely generated as a right ideal. Let $M$ be such an ideal and $D$ a simple subcoalgebra of $C$ such that $M=D^{\perp\left(C^{*}\right)}$. Arguing as in (ii) $\Rightarrow(\mathrm{i})$, we obtain that $C / D$ is finitely cogenerated. Thus $M$ is finitely generated.

Let $J \in \mathscr{F}_{C}$ and let $K$ be a two-sided ideal such that $K \in \mathscr{F}_{C}$ and $K \subset J$. Let $P_{1}, \ldots, P_{n}$ be maximal closed and cofinite two-sided ideals containing $J$ (there are only finitely many because $J$ is cofinite). Set $N=\cap_{i=1}^{n} P_{i}$, then $\operatorname{Rad}\left(C^{*} / K\right)=$ $N / K$. Hence, there is $n \in \mathbb{N}$ such that $N^{n} \subseteq K$. Let $H=P_{1} \cdots P_{n}$, then $H^{n} \subseteq N^{n} \subseteq$ $K \subset J$. The ideal $H$ is closed and cofinite by hypothesis, and finitely generated because the $P_{i}$ 's are so. Therefore, $J$ is finitely generated.

As an immediate consequence, we have the following corollary.

COROLlaRY 3.12. Let $C$ be a coalgebra such that $C_{1}$ is right Fr-Noetherian. The following assertions are equivalent:

(i) C has a right torsion rat functor;

(ii) $\mathscr{F}_{C}$ is closed under products;

(iii) $C$ is right $\mathscr{F}$-Noetherian.

We analyze in some cases the hypothesis of $C_{1}$ being right $\mathscr{F}$-Noetherian. Recall that a coalgebra $C$ is called almost connected if $C_{0}$ is finite dimensional.

Proposition 3.13. Let $C$ be an almost connected coalgebra. The following assertions are equivalent:

(i) $C_{1}$ is right $\mathscr{F}_{\text {-Noetherian; }}$

(ii) $C$ is locally finite;

(iii) $C_{n}$ is finite dimensional for all $n \in \mathbb{N}$. 
Proof. (i) $\Rightarrow$ (ii). If $C_{1}$ is right $\mathscr{F}$-Noetherian, then $C_{1}$ is locally finite. By [4, Theorem 2.4.5], $C$ is locally finite.

(ii) $\Rightarrow$ (iii). Since $C$ is locally finite, $C_{n}=C_{0} \wedge_{C} C_{n-1}$ is finite dimensional.

(iii) $\Rightarrow(\mathrm{i})$. It is clear since $C_{1}^{*}$ is finite dimensional.

COROLLARY 3.14. Let $C$ be an almost connected coalgebra. The following assertions are equivalent:

(i) C has a right torsion rat functor;

(ii) $\mathscr{F}_{C}$ is closed under products;

(iii) $C$ is locally finite;

(iv) $C$ is right $\mathscr{F}$-Noetherian.

Proof. It is sufficient to prove that (iii) $\Rightarrow$ (iv). Let $I$ be a finite-dimensional right coideal of $C$. There is $n \in \mathbb{N}$ such that $I \subseteq C_{n}$. For any $D \in \mathscr{Y}, I \wedge_{C} D \subseteq C_{n+1}$, which is finite dimensional. Setting $r=\operatorname{dim}\left(C_{n+1} / I\right)$, we have that $\operatorname{dim}\left(\left(I \wedge_{C}\right.\right.$ $D) / I) \leq r n_{D} \operatorname{dim}\left(S_{D}\right)$ for all $D \in \mathscr{S}$. From Lemma 3.10 and Proposition 3.8, $I^{\perp\left(C^{*}\right)}$ is finitely generated.

REMARK 3.15. (1) Corollary 3.14 is a generalization of [6, Corollary 21] where $C_{0}$ was assumed to be one dimensional. Notice that Corollary 3.14 is equivalent to [10, Theorem 4.6] and [2, Theorem 2.10].

(2) The hypothesis of $C$ being almost connected in Corollary 3.14 may be replaced by $C$ being a direct sum of almost connected coalgebras. This follows from the fact that a direct sum of coalgebras is right $\mathscr{F}$-Noetherian if and only if each term is so, see [10, Corollary 4.9] and [2, Proposition 2.8]. This includes the cocommutative case.

(3) Note that being locally finite does not depend on the right or left side. Thus, the statements of Corollary 3.14 are equivalent to their left versions.

In the pointed case, the upper bound of Lemma 3.10 takes a more transparent form. Let $C$ be a pointed coalgebra and $G(C)$ its set of group-like elements. For $x, y \in G(C)$, let $P_{x, y}(C)$ denote the space of $(x, y)$-primitive elements.

Proposition 3.16. Let $C$ be a pointed coalgebra. Then $C_{1}$ is right $F_{F}-$ Noetherian if and only if, for each $x \in G(C)$, the set $\left\{\operatorname{dim}\left(P_{x, y}(C)\right): y \in G(C)\right\}$ is bounded.

Proof. Assume that $C_{1}$ is right $\mathscr{F}$-Noetherian. Then $C_{1}$ is locally finite. Let $x \in G(C)$ and $M=(k x)^{\perp\left(C_{1}^{*}\right)}$. By hypothesis, $M$ is finitely generated. Proposition 3.8 yields that $C_{1} / k x$ is finitely cogenerated. For each $y \in G(C)$, the isotypic component of $k y$ in $C_{1} / k x$ is $\left(C_{1} / k x\right) \square_{C} k y \cong k y^{\left(n_{x, y}\right)}$ with $n_{x, y}=$ $\operatorname{dim}\left(P_{x, y}(C)\right)$. By hypothesis and Lemma 3.10, the set $\left\{n_{x, y}: y \in G(C)\right\}$ is bounded.

Conversely, let $D$ be a finite-dimensional subcoalgebra of $C_{1}$. We will show that $C / D$ is finitely cogenerated as a right comodule. For any $g, h \in G(C)$, let $P_{g, h}^{\prime}(C)$ be a subspace of $P_{g, h}(C)$ such that $P_{g, h}(C)=k(g-h) \oplus P_{g, h}^{\prime}(C)$. By the 
Taft-Wilson lemma [7, Theorem 5.4.1], $C_{1}=k G(C) \oplus\left(\oplus_{g, h \in G(C)} P_{g, h}^{\prime}(C)\right)$. We can write $D=\left(\oplus_{g \in F} k g\right) \oplus\left(\oplus_{g, h \in F} P_{g, h}^{\prime}(D)\right)$ with $F$ being a finite subset of $G(C)$. For each $g \in F$, set $D_{g}=k g \oplus\left(\oplus_{h \in F} P_{g, h}^{\prime}(D)\right)$. Then, $D=\oplus_{g \in F} D_{g}$ as right $C$ comodules. In order to prove that $D$ is finitely cogenerated, it suffices to prove that $D_{g}$ is so. The injective hull of $D_{g}$ is $E\left(D_{g}\right)=k g \oplus\left(\oplus_{h \in G(C)} P_{g, h}^{\prime}(C)\right)$. Now, $E\left(D_{g}\right) / D_{g} \cong \oplus_{h \in G(C)-F}(k h)^{\left(m_{g, h}\right)}$ with $m_{g, h}=\operatorname{dim}\left(P_{g, h}^{\prime}(C) / P_{g, h}^{\prime}(D)\right)$. From the hypothesis and Proposition 3.8, we deduce that $D_{g}$ is finitely cogenerated.

COROLLARY 3.17. Let $C$ be a pointed coalgebra satisfying that, for each $x \in$ $G(C)$, the set $\left\{\operatorname{dim}\left(P_{x, y}(C)\right): y \in G(C)\right\}$ is bounded. The following assertions are equivalent:

(i) C has a right torsion rat functor;

(ii) $\mathscr{F}_{\mathrm{C}}$ is closed under products;

(iii) $C$ is right $\mathscr{F}$-Noetherian.

We finish this paper by constructing new examples of right $\mathscr{F}$-Noetherian coalgebras. Combining Example 3.7(ii) and Theorem 3.11, we have the following corollary.

COROLlaRY 3.18. Let $C$ be a coalgebra. If $C_{n}$ is left semiperfect for all $n \in \mathbb{N}$, then $C$ is right $\mathscr{F}$-Noetherian.

EXAMPLE 3.19. For a quiver $\Gamma$, the path coalgebra $k \Gamma$ is the $k$-vector space generated by the paths in $\Gamma$ with comultiplication $\Delta$ and counit $\varepsilon$ defined by

$$
\Delta(\gamma)=\sum_{\alpha \beta=\gamma} \alpha \otimes \beta, \quad \varepsilon(\gamma)= \begin{cases}0, & \text { if }|\gamma|>0, \\ 1, & \text { if }|\gamma|=0,\end{cases}
$$

where $\alpha, \beta$, and $\gamma$ are paths, $\alpha \beta$ is the concatenation of paths, and $|\cdot|$ denotes the length of a path. Assume that, for every vertex $v \in \Gamma$ and any $n \in \mathbb{N}$, there is a finite number of paths of length less than or equal to $n$ ending at $v$. This condition assures that $(k \Gamma)_{n}$ is left semiperfect for all $n \in \mathbb{N}$. Then, the path coalgebra $k \Gamma$ is right $\mathscr{F}$-Noetherian.

ACKNOWLEDGment. This research was supported by Grant BFM2002-02717 from DGES.

\section{REFERENCES}

[1] E. Abe, Hopf Algebras, Cambridge University Press, Cambridge, 1977.

[2] J. Cuadra, C. Năstăsescu, and F. Van Oystaeyen, Graded almost Noetherian rings and applications to coalgebras, J. Algebra 256 (2002), no. 1, 97-110.

[3] J. A. Green, Locally finite representations, J. Algebra 41 (1976), no. 1, 137-171.

[4] R. G. Heyneman and D. E. Radford, Reflexivity and coalgebras of finite type, J. Algebra 28 (1974), 215-246.

[5] B. I.-P. Lin, Products of torsion theories and applications to coalgebras, Osaka J. Math. 12 (1975), no. 2, 433-439.

[6] _ Semiperfect coalgebras, J. Algebra 49 (1977), no. 2, 357-373. 
[7] S. Montgomery, Hopf Algebras and Their Actions on Rings, CBMS Regional Conference Series in Mathematics, vol. 82, American Mathematical Society, Washington, DC, 1993.

[8] D. E. Radford, Coreflexive coalgebras, J. Algebra 26 (1973), 512-535.

[9] _ On the structure of ideals of the dual algebra of a coalgebra, Trans. Amer. Math. Soc. 198 (1974), 123-137.

[10] T. Shudo, A note on coalgebras and rational modules, Hiroshima Math. J. 6 (1976), no. 2, 297-304.

[11] B. Stenström, Rings of Quotients. An Introduction to Methods of Ring Theory, Die Grundlehren der Mathematischen Wissenschaften, vol. 217, SpringerVerlag, New York, 1975.

[12] M. E. Sweedler, Hopf Algebras, Mathematics Lecture Note Series, W. A. Benjamin, New York, 1969.

[13] M. Takeuchi, Morita theorems for categories of comodules, J. Fac. Sci. Univ. Tokyo Sect. IA Math. 24 (1977), no. 3, 629-644.

J. Cuadra: Departamento de Álgebra y Análisis Matemático, Universidad de Almería, 04120 Almería, Spain

E-mail address: jcdiaz@ua1.es 


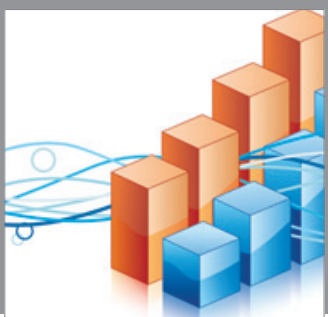

Advances in

Operations Research

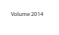

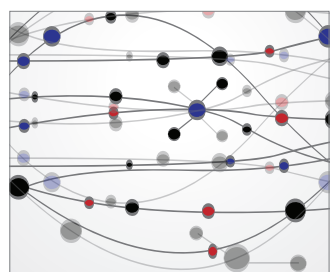

\section{The Scientific} World Journal
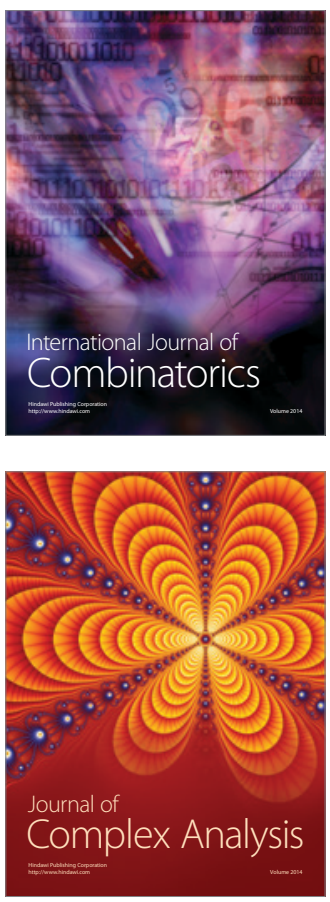

International Journal of

Mathematics and

Mathematical

Sciences
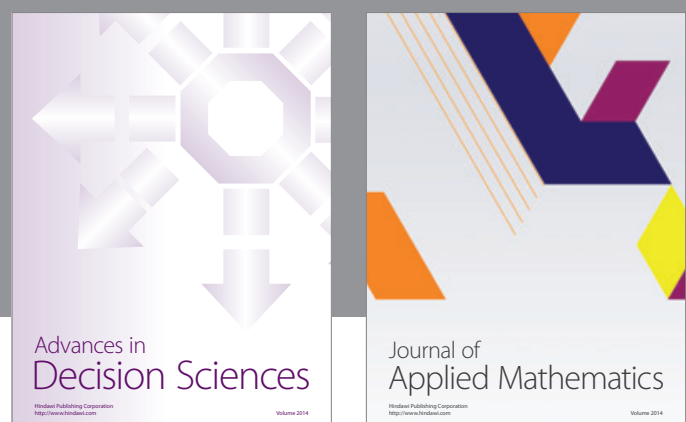

Journal of

Applied Mathematics
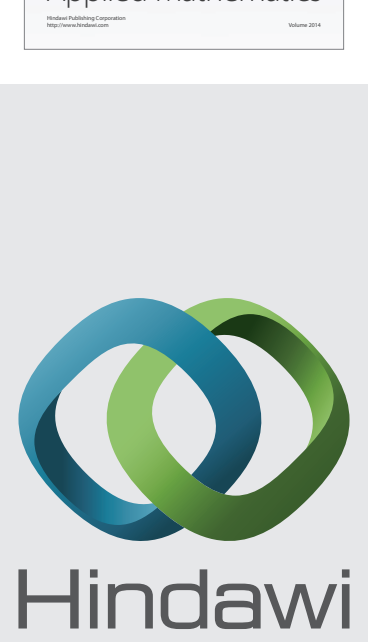

Submit your manuscripts at http://www.hindawi.com
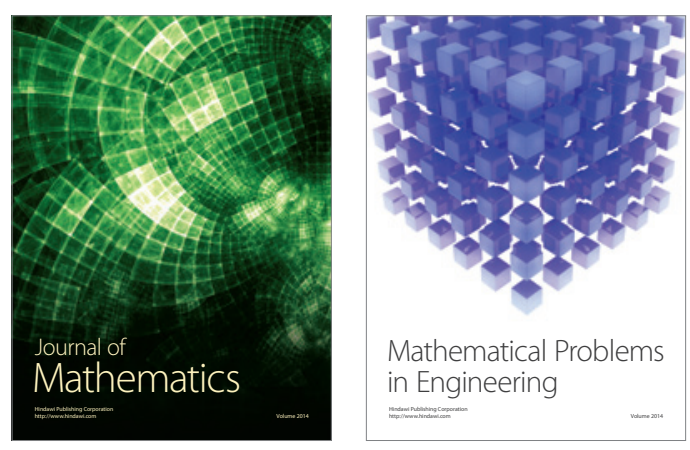

Mathematical Problems in Engineering
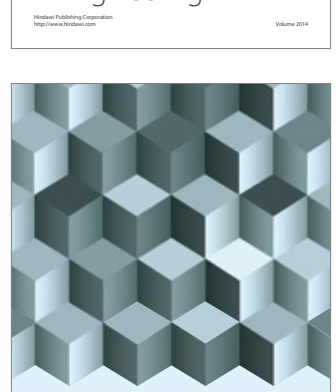

Journal of

Function Spaces
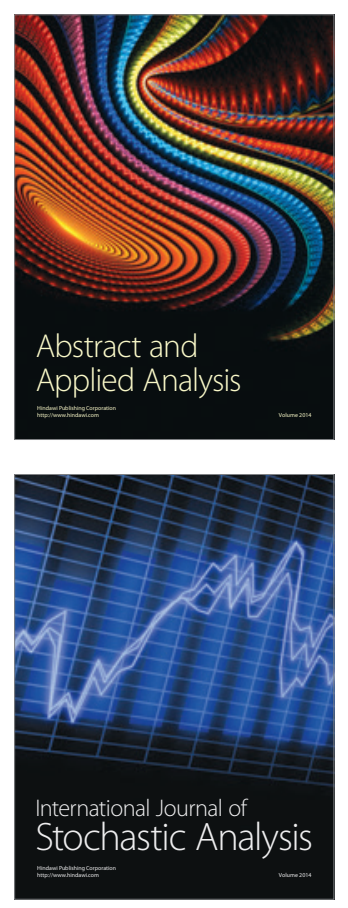

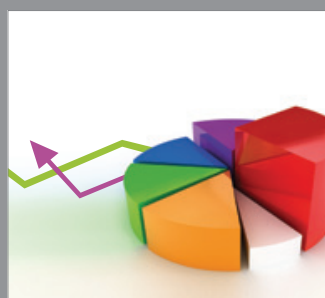

ournal of

Probability and Statistics

Promensencen
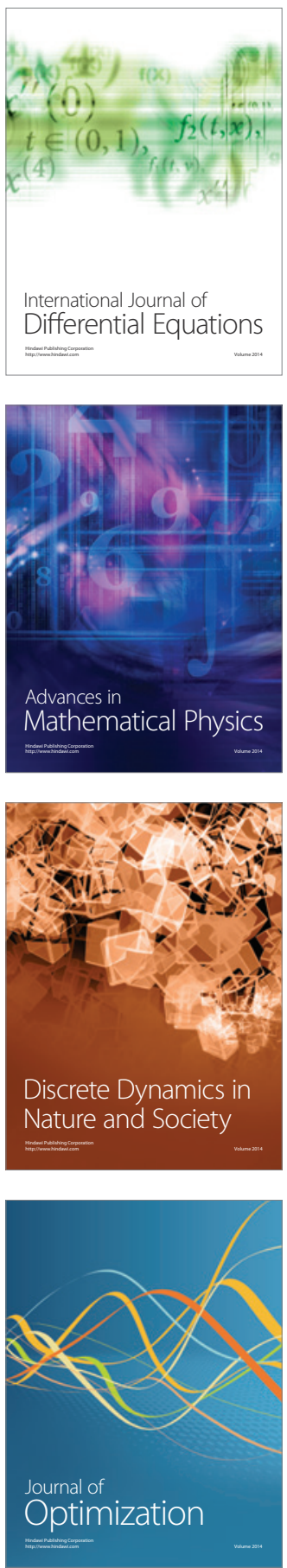